\title{
Improvement of data refresh rate for dual serial port to universal serial bus acquisition system
}

\begin{abstract}
Before the Universal Serial Bus (USB) Technologies has become popular, there are a lot of software and hardware computer peripheral development based on traditional external interface like General Purpose Interface Bus (GPIB- IEEE488), parallel port, serial port and $\mathrm{PS} / 2$. Developing these custom software and hardware computer peripheral is less difficult as compared to peripheral that is designed based on USB. The main reason for the difficulty is that the USB is designed to be user friendly but not developer friendly. For that reason, many developers will maintain using the traditional port when designing their custom application. To support traditional interface on newer PC with only USB port, there are devices in the market to convert the old external port to USB. One of the converters is the USB to dual serial converter. In the market, there are products which are readily available. However, it lacks of certain capability such as the ability to access serial port simultaneously which reduce the data refresh rate. The main purpose of this paper is to discuss technique in designing a USB to serial converter with capability of connecting two serial devices which is accessible simultaneously to improve the data refresh rate.
\end{abstract}

Keyword: Component; USB; Serial port; Data acquisition 